\title{
AQP2 as a diagnostic immunohistochemical marker for pheochromocytoma and/or paraganglioma
}

\author{
Dongfeng Niu", Yanhua Bai", Qian Yao, Lixin Zhou, Xiaozheng Huang, Chen Zhao \\ Department of Pathology, Key Laboratory of Carcinogenesis and Translational Research (Ministry of Education), Peking University Cancer Hospital \\ \& Institute, Beijing 100142, China \\ Contributions: (I) Conception and design: D Niu, Y Bai; (II) Administrative support: D Niu, L Zhou; (III) Provision of study materials or patients: Y \\ Bai, L Zhou, X Huang, C Zhao; (IV) Collection and assembly of data: D Niu, Q Yao; (V) Data analysis and interpretation: D Niu, Y Bai, Q Yao; (VI) \\ Manuscript writing: All authors; (VII) Final approval of manuscript: All authors. \\ \#These authors contributed equally to this work. \\ Correspondence to: Dongfeng Niu, MD, PhD. Peking University Cancer Hospital \& institute, No. 52, Fucheng Road, Haidian District, Beijing \\ 100142, China. Email: Dongfengniu@foxmail.com.
}

\begin{abstract}
Background Aquaporin2 (AQP2) is water channel protein that is widely distributed among mammalian tissues and plays a major role in water homeostasis. However, little is known about the expression and significance of AQP2 in human adrenal tumors. Thus, we performed an immunohistochemical investigation of AQP2 in normal and neoplastic adrenal tissues.

Methods: AQP2 protein expression was investigated in 190 adrenal tumor patients using immunohistochemistry. Correlation between protein expression and clinicopathological features was statistically analyzed.
\end{abstract}

Results: We demonstrated immunopositivity for AQP2 in all adrenal medulla-originating tumors, including 96 pheochromocytomas (PCC), 7 metastatic pheochromocytomas (MPCC), and 10 neuroblastic tumors (NT) and 13 extra-adrenal paragangliomas (EAPGL). Whereas, APQ2 was absent from the 52 adrenal cortical adenomas and 2 adrenal cortical carcinomas examined. The 10 metastatic carcinomas examined in adrenal tissue were also negative for AQP2. In 82 (85\%) of the 96 samples from patients with PCC, we studied the relationship between clinicopathologic factors and AQP2 expression and our findings suggested that the tumors that exhibited diffuse expression pattern of AQP2 were larger in diameter than those exhibiting focal $(\mathrm{P}=0.007)$ or mediate expression pattern $(\mathrm{P}=0.001)$.

Conclusions: AQP2 protein is significantly expressed in normal adrenal medullary tissues and medullary tumors (including PCC, MPCC and NT) as well as EAPGL. AQP2 expression may indicate the origin of normal adrenal tissues, and its expression in cancer tissue may reflect the maintenance of water metabolism via AQP2 during tumorigenesis. AQP2 may serve as a valuable marker for the differential diagnosis of adrenal tumors.

Koywords: Aquaporin2 (AQP2); immunohistochemistry; pheochromocytoma (PCC); paraganglioma

Submitted Sep 23, 2019. Accepted for publication Jan 04, 2020.

doi: $10.21037 /$ gs.2020.01.19

View this article at: http://dx.doi.org/10.21037/gs.2020.01.19

\section{Introduction}

Primary adrenal tumors comprise two main distinct types. Adrenal cortical tumors are derived from the cortex and mainly include adrenal cortical adenoma and cortical carcinoma. Adrenal tumors originating from the medulla contain pheochromocytoma (PCC) and neuroblastic tumors (NT). PCC and extra-adrenal paraganglioma (EAPGL) are both the catecholamine-secreting neuroendocrine tumors while the latter arise from the extra-adrenal paraganglia. 
Table 1 Summary of immunohistochemistry for AQP2 in adrenal gland tumors

\begin{tabular}{|c|c|c|c|c|}
\hline Tumor types & \multicolumn{4}{|c|}{ AQP2 expression } \\
\hline \multicolumn{5}{|l|}{ Adrenal cortical tumor } \\
\hline Adrenal cortical adenoma $(n=52)$ & $52(100 \%)$ & $0(0 \%)$ & $0(0 \%)$ & $0(0 \%)$ \\
\hline Adrenal cortical carcinoma $(n=2)$ & $2(100 \%)$ & $0(0 \%)$ & $0(0 \%)$ & $0(0 \%)$ \\
\hline Pheochromocytoma $(n=96)$ & $0(0 \%)$ & $5(5 \%)$ & $10(10 \%)$ & $81(85 \%)$ \\
\hline Metastatic pheochromocytoma $(n=7)$ & $0(0 \%)$ & $1(14 \%)$ & $2(29 \%)$ & $4(57 \%)$ \\
\hline Neuroblastic tumor $(n=10)$ & $6(60 \%)$ & $4(40 \%)$ & $0(0 \%)$ & $0(0 \%)$ \\
\hline Extra-adrenal paraganglioma $(n=13)$ & $0(0 \%)$ & $0(0 \%)$ & $0(0 \%)$ & $13(100 \%)$ \\
\hline
\end{tabular}

0 , negative; $1+$, focal ( $1 \%$ to $9 \%$ ); $2+$, intermediate (10\% to $50 \%$ ); $3+$, diffuse (more than $50 \%$ ).

About $40 \%$ of PCCs and EAPGLs are identified as inherited forms due to the germline mutation in susceptibility genes such as $V H L, S D H B, S D H D$ and $S D H C$ genes. The remaining $60 \%$ are thought to be sporadic cases. At least one-third of the sporadic tumors contain a somatic mutation in predisposing gene including $V H L, R E T, N F 1$ and $\operatorname{HRAS}(1,2)$.

The aquaporins (AQPs) are a family of small membrane proteins $(25 \sim 34 \mathrm{kDa})$ of 13 subtypes (AQP0-AQP12) that serve as hydrophobic and integral membrane channel proteins that facilitate rapid passive movement of water (3). AQPs not only play important roles in fluid transport in many epithelial and endothelial tissues, but they also participate in the regulation of important cellular functions, including cell proliferation and migration, apoptosis, phagocytosis and neuronal signal transduction (4). In neoplastic tissues, AQP subtypes were involved in invasion, migration, and proliferation of tumors in several organs, including skin, urinary bladder, stomach, colon, kidney (5-8) and so on. Among these subtypes, AQP2 is a classical water channel protein that regulates water homeostasis in normal tissue (9), and its expression has been demonstrated in the kidney cortex/medulla, gastric pit, small intestine, pancreatic islet, and fallopian tubes, among other tissues (10). AQP2 is responsible for regulating the volume and osmolarity of urine in the kidney (10) and involves pain transmission in the trigeminal nerve (4). Recently, the expression of AQP2 was reported in endometrial carcinoma and brain glioma, where it mediates estrogen-induced cell migration and invasion $(11,12)$. Very limited information is available concerning the role of AQP2 in human adrenal tumors.

In the current study, we performed immunohistochemical analysis of AQP2 expression in 190 adrenal tumor samples. Our findings implicated AQP2 as a valuable indicator for differential diagnosis of adrenal tumors.

\section{Methods}

\section{Cases selection}

We selected a series of surgical specimens of neoplastic adrenal tissues (190 cases), which were consecutively collected between 1993 and 2012 from Peking University Cancer Hospital \& Institute. The cases, summarized in Table 1, included adrenal cortical adenoma $(\mathrm{n}=52)$, adrenal cortical carcinoma $(n=2)$, PCC $(n=96)$, metastatic pheochromocytoma (MPCC) $(\mathrm{n}=7)$, EAPGL $(\mathrm{n}=13)$, NT $(\mathrm{n}=10)$, and metastatic carcinoma $(\mathrm{n}=10)$. We also investigated normal tissue adjacent to the tumors. Hematoxylin-eosin (HE) staining was carried out on all of the slides, and the corresponding diagnosis was made on the basis of the World Health Organization classification criteria (13). All MPCC cases had metastasized to lymphatic nodes and/or several organs including liver, lung, pancreas, and colon. All patients with metastatic carcinoma had a history of primary cancer, including 2 hepatocellular carcinomas, 2 renal cell carcinomas, 3 pulmonary carcinomas, 1 breast carcinoma, 1 
rectal carcinoma and 1 gastric carcinoma. Clinicopathologic factors including age, sex, and tumor size were examined in $82(85 \%)$ of the 96 patients with PCC. The study protocol was approved by the institutional ethics committee of the Peking University Cancer Hospital \& Institute (No. 2014KT196).

\section{Immunobistochemical assay}

Immunohistochemical analysis was performed on $3-\mu \mathrm{m}$ sections of formalin-fixed and paraffin-embedded tissues mounted on silanized slides. The deparaffinized sections were placed in plastic Coplin jars filled with $10 \mathrm{mmol} / \mathrm{L}$ citrate buffer $(\mathrm{pH} 6.0)$ and incubated for 10 minutes at $120{ }^{\circ} \mathrm{C}$ in an autoclave for heat antigen retrieval. After the autoclave treatment, endogenous peroxidase was quenched

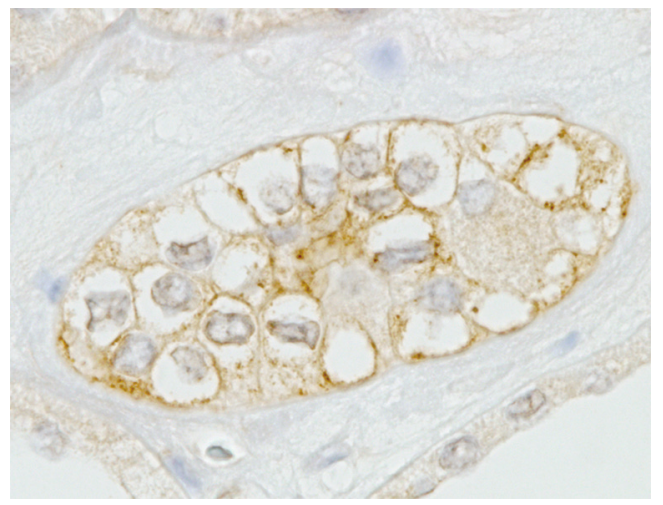

Figure 1 Immunohistochemical staining of AQP2 in normal kidney tissue for positive control. The cytoplasmic membrane of collecting ducts presents immunopositive for AQP2. (Magnification: $\times 600)$. with $3 \%$ (vol/vol) $\mathrm{H}_{2} \mathrm{O}_{2}$ for 10 minutes. The sections were incubated with an anti-AQP2 antibody (1:1,000 dilution, Sigma, USA) at room temperature for 1 hour. We used normal tissue from the collecting duct of the kidney as a positive control and omitted the primary antibody for the negative control. We quantified the immunoreactivity of AQP2 using a four-tier scale from 0 to $3+$ : $0=$ no staining, $1+=$ focal $(1 \%$ to $9 \%$ positive cells), $2+=$ intermediate $(10 \%$ to $50 \%$ positive cells), $3+=$ diffuse $(50 \%$ or more positive cells). Immunohistochemical staining was analyzed by two observers (DN and $\mathrm{YB}$ ) blinded to all clinicopathological information. Discrepancies in immunohistochemical analysis were reconciled following the discussion of 2 observers.

\section{Statistics}

The clinicopathologic characteristics were statistically analyzed by ANOVA (age, tumor size, and tumor weight versus AQP2 positive pattern) and multiple comparison tests (sex versus AQP2 positive pattern). We set statistical significance at $\mathrm{P}<0.05$ and performed data analyses with SPSS V.25.0 (SPSS, Chicago, Illinois, USA).

\section{Results}

\section{AQP2 in normal tissues}

We chose normal kidney tissues as a positive control. As expected, AQP2 protein was localized specifically in the membrane of the collecting duct cells of the kidney (Figure 1). Then we studied the immunostaining of AQP2 in adrenal tissue and observed immunopositivity of AQP2 in cells of the adrenal medulla rather than in adrenal cortical cells (Figure 2).
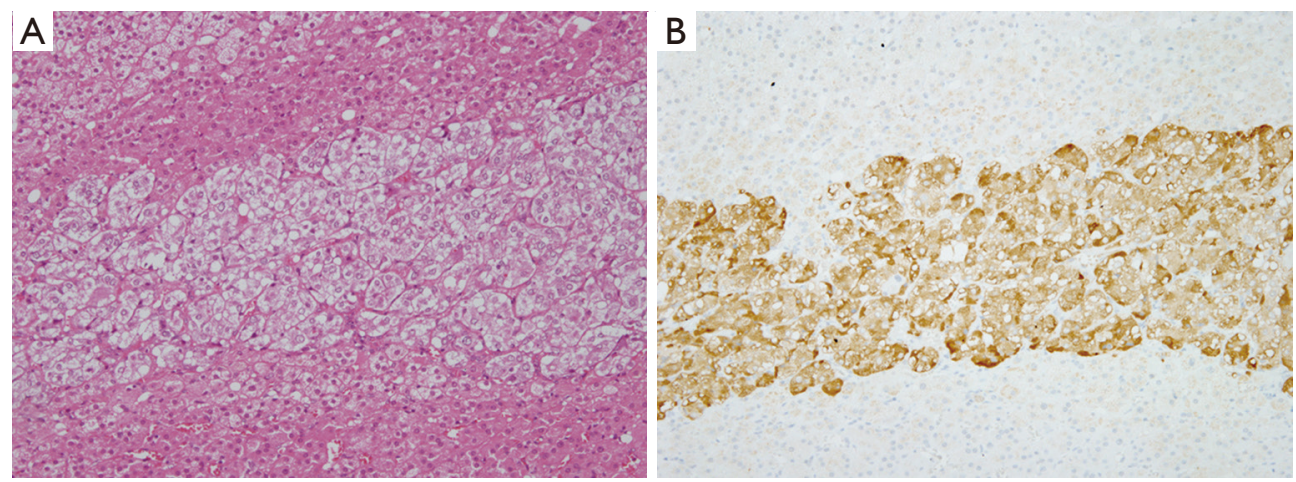

Figure 2 Immunohistochemical staining for AQP2 in normal adrenal tissues/cells. (A) Normal adrenal medullar cells and cortical cells is observed (H\&E, magnification: $\times 200)$; (B) diffuse immunopositive for AQP2 is showed in adrenal medullar cells, but not in cortical cells (magnification: $\times 200)$. 

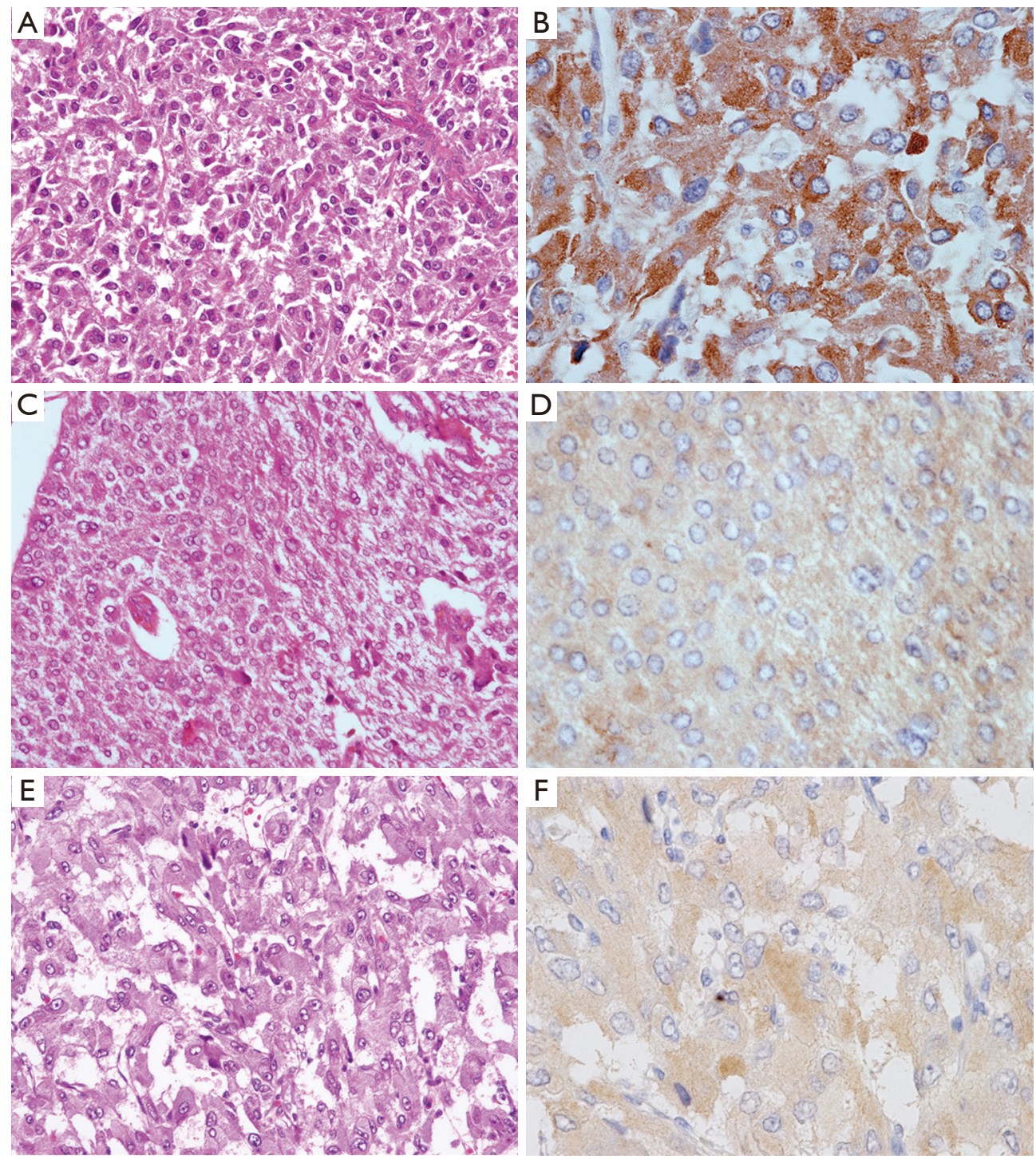

Figure 3 Immunohistochemical staining for AQP2 in PCC. PCC shows zellballen (A), solid architecture (C), and intracytoplasmic oncocytic feature (E) (H\&E, magnification: ×200). The corresponding immunostaining of AQP2 in the PCC cells (B,D,F) (magnification: $\times 400)$.

\section{AQP2 expression in adrenal PCC and EAPGLs}

The immunohistochemical results of AQP2 for adrenal PCC and EAPGLs are summarized in Table 1. We found that AQP2 protein was expressed in all adrenal medullary tumors and EAPGLs. Among 96 PCCs, most tumors exhibited a diffuse pattern of AQP2 expression, identified in $85 \%$ (81/96) of cases, while focal expression and intermediate expression were observed in only $5 \%(5 / 96)$ and $10 \%(10 / 96)$ of cases, respectively. Immunohistochemical intensity of AQP2 in PCC tumors with Zellballen architecture (Figure $3 A, B$ ) was notably strong compared to tumors with solid architecture (Figure $3 C, D)$. In MPCC tissues, the distribution of AQP2-positive cells varied: $57 \%(4 / 7)$ in diffuse, $29 \%(2 / 7)$ in intermediate and $14 \%(1 / 7)$ in focal tumor tissues (Figure $4 A, B, C, D, E, F)$. The focal expression pattern of AQP2 was identified in $4(40 \%)$ of 10 Schwannian stroma cells of NTs. All 13 EAPGLs exhibited a diffuse distribution of AQP2.

\section{AQP2 expression pattern in adrenal cortical tumors and other adrenal tumors}

We found that AQP2 was not expressed in the adrenal 

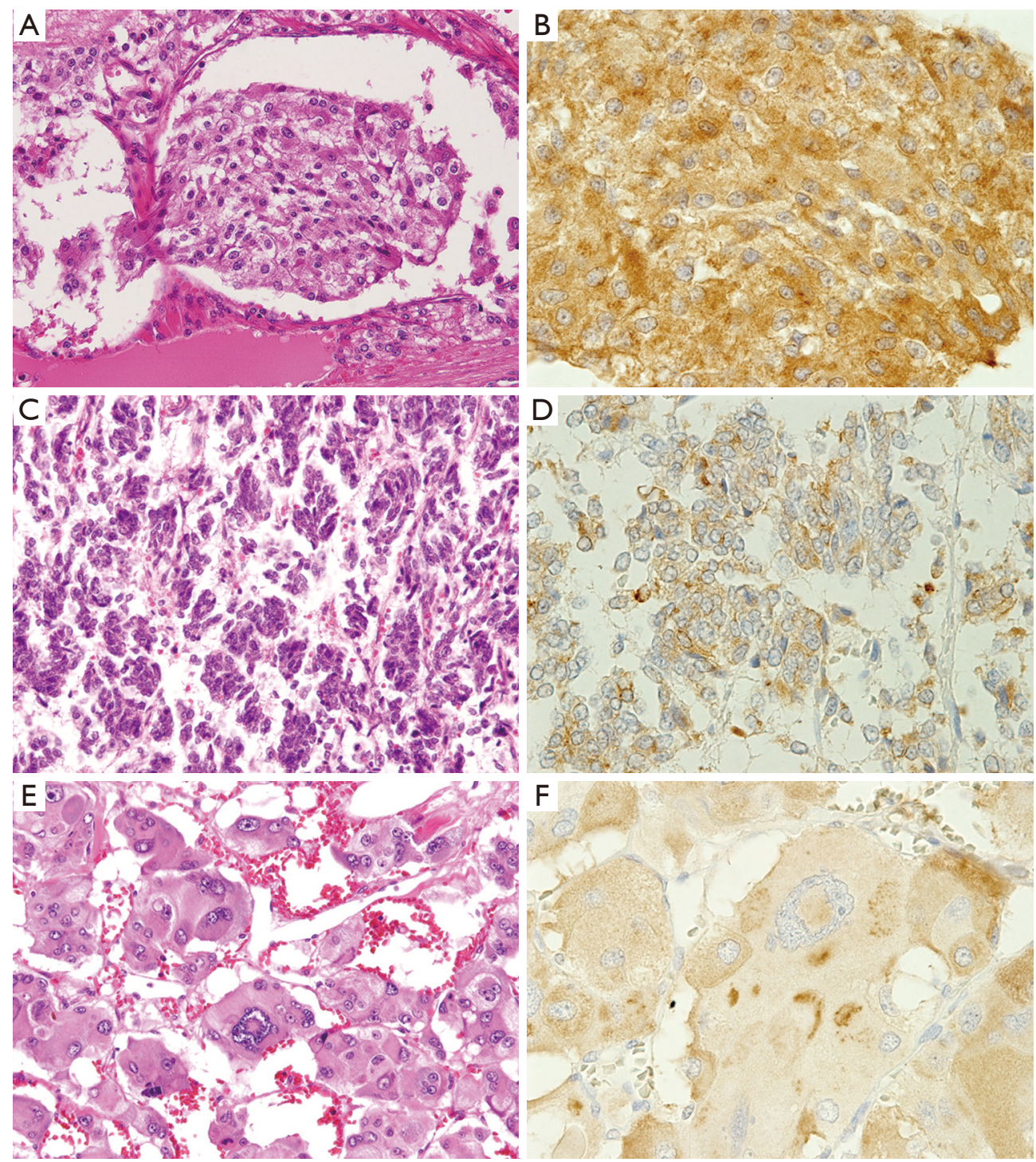

Figure 4 Immunohistochemical staining for AQP2 in MPCC. MPCC exhibits vascular invasion (A), nest growth pattern (C), and bizarre nuclear feature $(\mathrm{E})(\mathrm{H} \& \mathrm{E}$, magnification: $\times 200)$. AQP2 protein is seen in the MPCC cells $(\mathrm{B}, \mathrm{D}, \mathrm{F})($ magnification: $\times 400)$.

cortical tumors, which included 52 cortical adenomas and 2 cortical carcinomas (Figure $5 A, B, C, D$ ). All 10 metastatic carcinomas were also negative for $\mathrm{AQP} 2$ protein.

\section{The association between clinicopathologic factors and AQP2 expression in adrenal PCCs}

The patients with PCC comprised 44 women and 38 men, ranging from 29 to 83 years in age (median, 50). The tumor diameter ranged from 1.1 to $45 \mathrm{~cm}$ (median, 5). The tumor weight ranged from 5 to $740 \mathrm{~g}$ (median, 45). The tumors showing the diffuse expression pattern of AQP2 were larger in diameter than those with a focal $(\mathrm{P}=0.007)$ or mediate expression pattern $(\mathrm{P}=0.001)$. Besides, there were no significant effects of other clinicopathologic factors (age, sex, and tumor weight) on the expression of AQP2. All data was showed in Table 2.

\section{Discussion}

AQP2 is a typical water channel protein that plays an essential role in fluid homeostasis in normal tissues. AQP2 


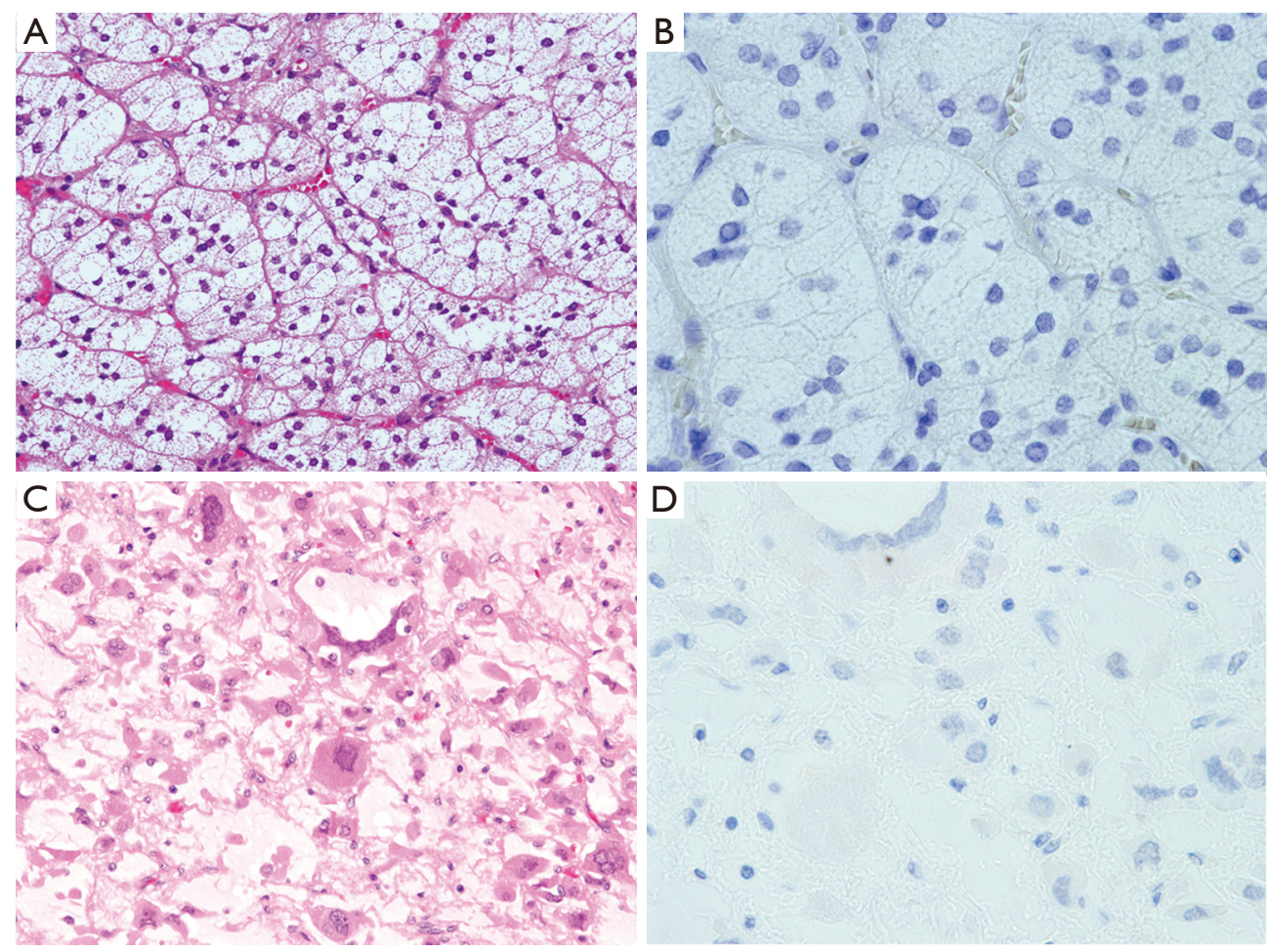

Figure 5 Immunohistochemical staining for AQP2 in adrenal cortical tumor tissues. Adrenal cortical adenoma presents microvesicle or clear cytoplasm mimicking the zona fasciculata (A). Adrenal cortical carcinoma shows severe nuclear atypia, and interstitial myxoid change (C) (H\&E, magnification: ×200). The tumor tissues/cells are negative for AQP2 (B,D, magnification: ×400).

Table 2 Clinicopathologic factors and their relation with AQP2 expression

\begin{tabular}{lccr}
\hline Clinicopathologic factors & \multicolumn{1}{c}{ AQP2 } & $3+$ \\
\hline Age (years) & $1+$ & $51.8 \pm 11.3$ & $52.7 \pm 14.0$ \\
Sex & $50.4 \pm 11.3$ & & 7.1 \\
Male $(\mathrm{n}=38)(\%)$ & 4.8 & 10.4 & 88.1 \\
Female $(\mathrm{n}=44)(\%)$ & 6.3 & $5.8 \pm 2.6$ & 83.3 \\
Tumor size $(\mathrm{cm})$ & $6.1 \pm 5.6$ & $68.0 \pm 51.1$ & $17.4 \pm 16.0^{* *}$ \\
Tumor weight $(\mathrm{g})$ & $48.9 \pm 38.7$ & $101.0 \pm 72.6$ & \\
\hline
\end{tabular}

0 , negative; $1+$, focal ( $1 \%$ to $9 \%$ ); $2+$, intermediate (10\% to $50 \%$ ); $3+$, diffuse (more than $50 \%$ ). Statistical significance was calculated using the ANOVA test (age, size, and weight) and multiple comparison (sex). ${ }^{*}, \mathrm{P}<0.01$.

was originally identified in the renal collecting duct cells, where it functions as the vasopressin-sensitive water channel (14-16). Its expression also has been reported in pancreatic islets, fallopian tubes, peripheral/central nerves, and gastrointestinal tissues (10).

To our knowledge, the expression of AQP2 has not been reported in normal adrenal gland tissues. In this study, we used immunohistochemical analysis to show that AQP2 protein was expressed in normal adrenal medullary cells but not in adrenal cortical cells. It has been confirmed that AQP2 is linked to vasopressin-stimulated water reabsorption in kidney (17). From this point of view, it is 
conceivable that AQP2 in adrenal medullar cells may play an important role in water homeostasis and other biological functions. However, its expression also raises two questions: (I) is AQP2 the only AQP subtype with a functional role in adrenal medullar cells; and (II) which subtypes of AQPs are responsible for water transport in adrenal cortical cells? We previously demonstrated that AQP3 might play a role in fluid homeostasis in medullary thyroid carcinoma (18) but not in adrenal gland tumors (19). However, additional studies are needed to further elucidate these questions. Moreover, in embryology, the medulla and cortex of the adrenal gland have distinct embryonic origins. Medullary cells originate from the neuroectoderm, whereas cortical cells originate from the mesoderm. Thus, our findings also suggested that AQP2 expression may reflect the origin of normal adrenal gland tissues.

A lack of function and/or reduced expression of AQP2 has been observed in several diseases, such as renal failure (20), acquired nephrogenic diabetes insipidus, and postobstructive polyuria (21). AQP2 can also induce the cellular migration and invasion of endometrial carcinoma (11). We demonstrated that AQP2 was specially expressed in all adrenal medulla-originating tumors but not in corticaloriginating tumors. These findings suggested that AQP2 may be associated with the biological metabolism of water in PCCs. However, we failed to find any difference on AQP2 staining between PCC and MPCC, which implied that AQP2 may not be served as a valuable predictive parameter on metastasis disease. According to our findings, the immunohistochemical staining of AQP2 can be used as helpful diagnostic markers to distinguish PCCs and paraganglioma from other adrenal tumors such as adrenal cortical tumors and metastatic tumors for routine pathologic diagnosis, similar to the neural markers chromograninA (13), among others. AQP2 expression in normal adrenal medullar tissues and in adrenal medulla-originating tumors also implies that the water metabolism function of AQP2 is well maintained during tumorgenesis.

We analyzed the correlation between AQP2 expression and clinicopathologic factors in adrenal PCCs. The results showed that tumors with the diffuse expression pattern of AQP2 were larger in diameter than those displaying focal or mediate expression patterns. AQP subtypes play important roles in cell growth and proliferation in several types of cancer, such as squamous cell carcinomas $(22,23)$, breast carcinomas (24), ovarian carcinomas (25), and brain tumors (26). Thus, it is reasonable to suggest that AQP2 may be associated with the growth and/or proliferation of PCCs, and the present findings confirmed our speculation that AQP2 participates in the biological metabolism of water in the tumors. Because of the limited cases of MPCC, we failed to compare the AQP2 expression and clinicopathologic factors between PCC and MPCC. In addition, we only analyzed AQP2 immunohistochemical expression in adrenal tumors, but the AQP family comprises 13 subtypes with differential expression patterns in normal tissues. Different combinations of these various types of AQPs may be more valuable for diagnosis of adrenal tumors, warranting further studies.

In conclusion, the AQP2 protein is expressed in normal adrenal medullary tissues and in medullar tumors (PCC, MPCC and NT) as well as in EAPGL, but it is not found in normal adrenal cortical tissue or in cortical tumors. Thus, AQP2 may have differential diagnostic value in identifying adrenal tumors. In addition, PCCs with a diffuse pattern of AQP2 expression were larger in diameter than those displaying focal or mediate expression. Our findings also suggest that AQP2 expression may reflect the origin of normal adrenal gland tissues. AQP2 not only participates in the biological metabolism of water in PCC but also likely maintains this function during tumorgenesis in adrenal medullar tumors.

\section{Acknowledgments}

Funding: This study was supported by grants from the National Natural Science Foundation of China (No. 81301879 and 81202114).

\section{Footnote}

Conflicts of Interest: All authors have completed the ICMJE uniform disclosure form (available at http://dx.doi. org/10.21037/gs.2020.01.19). The authors have no conflicts of interest to declare.

Ethical Statement: The authors are accountable for all aspects of the work in ensuring that questions related to the accuracy or integrity of any part of the work are appropriately investigated and resolved. The research program was approved by the ethics committee of Peking University Cancer Hospital \& Institute (No. 2014KT196). 
Open Access Statement: This is an Open Access article distributed in accordance with the Creative Commons Attribution-NonCommercial-NoDerivs 4.0 International License (CC BY-NC-ND 4.0), which permits the noncommercial replication and distribution of the article with the strict proviso that no changes or edits are made and the original work is properly cited (including links to both the formal publication through the relevant DOI and the license). See: https://creativecommons.org/licenses/by-nc-nd/4.0/.

\section{References}

1. Favier J, Amar L, Gimenez-Roqueplo AP. Paraganglioma and phaeochromocytoma: from genetics to personalized medicine. Nat Rev Endocrinol 2015;11:101-11.

2. Karasek D, Frysak Z, Pacak K. Genetic testing for pheochromocytoma. Curr Hypertens Rep 2010;12:456-64.

3. Agre P, Kozono D. Aquaporin water channels: molecular mechanisms for human diseases. Febs Lett 2003;5 55:72-8.

4. Ma T, Gao H, Fang X, et al. Water channel proteins in the peripheral nervous system in health and disease. Mol Aspects Med 2012;33:605-11.

5. Moosavi MS, Elham Y. Aquaporins 1, 3 and 5 in Different Tumors, their Expression, Prognosis Value and Role as New Therapeutic Targets. Pathol Oncol Res 2019. [Epub ahead of print].

6. Rubenwolf $\mathrm{P}$, Thomas C, Denzinger S, et al. Loss of AQP3 protein expression is associated with worse progressionfree and cancer-specific survival in patients with muscleinvasive bladder cancer. World J Urol 2015;33:1959-64.

7. Nico B, Ribatti D. Aquaporins in tumor growth and angiogenesis. Cancer Lett 2010;294:135-8.

8. Kafé H, Verbavatz JM, Cochand-Priollet B, et al. Collecting duct carcinoma: an entity to be redefined? Virchows Arch 2004;445:637-40.

9. Tajika Y, Matsuzaki T, Suzuki T, et al. Aquaporin-2 is retrieved to the apical storage compartment via early endosomes and phosphatidylinositol 3-kinase-dependent pathway. Endocrinology 2004;145:4375-83.

10. Mobasheri A, Wray S, Marples D. Distribution of AQP2 and AQP3 water channels in human tissue microarrays. J Mol Histol 2005;36:1-14.

11. Zou LB, Zhang RJ, Tan YJ, et al. Identification of estrogen response element in the aquaporin-2 gene that mediates estrogen-induced cell migration and invasion in human endometrial carcinoma. J Clin Endocrinol Metab
2011;96:E1399-408.

12. Wan S, Jiang J, Zheng C, et al. Estrogen nuclear receptors affect cell migration by altering sublocalization of AQP2 in glioma cell lines. Cell Death Discov 2018;4:49.

13. Lloyd RV, Osamura RY, Kloppel G, et al. WHO classification of tumours: pathology and genetics of tumours of endocrine organs. 4th edition. Lyon: IARC, 2017.

14. Ranieri M, Di Mise A, Tamma G, et al. Vasopressinaquaporin-2 pathway: recent advances in understanding water balance disorders. F1000Res 2019. doi: http://dx.doi. org/10.12688/f1000research.16654.1.

15. Sasaki S, Fushimi K, Saito H, et al. Cloning, characterization, and chromosomal mapping of human aquaporin of collecting duct. J Clin Invest 1994;93:1250-6.

16. Kortenoeven ML, Fenton RA. Renal aquaporins and water balance disorders. Biochim Biophys Acta 2014;1840:1533-49.

17. Wilke C, Sheriff S, Soleimani M, et al. Vasopressinindependent regulation of collecting duct aquaporin-2 in food deprivation. Kidney Int 2005;67:201-16.

18. Niu D, Kondo T, Nakazawa T, et al. Differential expression of aquaporins and its diagnostic utility in thyroid cancer. Plos One 2012;7:e40770.

19. Niu D, Kondo T, Nakazawa T, et al. Expression of aquaporin 3 in human neoplastic tissues. Histopathology 2012;61:543-51.

20. Dos Santos IF, Sheriff S, Amlal S, et al. Adenine acts in the kidney as a signaling factor and causes salt- and water-losing nephropathy: early mechanism of adenineinduced renal injury. Am J Physiol Renal Physiol 2019;316:F743-57.

21. Marples D, Christensen BM, Frokiaer J, et al. Dehydration reverses vasopressin antagonist-induced diuresis and aquaporin-2 downregulation in rats. Am J Physiol 1998;275:F400-9.

22. Kusayama M, Wada K, Nagata M, et al. Critical role of aquaporin 3 on growth of human esophageal and oral squamous cell carcinoma. Cancer Sci 2011;102:1128-36.

23. Ishimoto S, Wada K, Usami Y, et al. Differential expression of aquaporin 5 and aquaporin 3 in squamous cell carcinoma and adenoid cystic carcinoma. Int J Oncol 2012;41:67-75.

24. Shi Z, Zhang T, Luo L, et al. Aquaporins in human breast cancer: identification and involvement in carcinogenesis of breast cancer. J Surg Oncol 2012;106:267-72. 
25. Yan C, Yang J, Shen L, et al. Inhibitory effect of Epigallocatechin gallate on ovarian cancer cell proliferation associated with aquaporin 5 expression. Arch Gynecol Obstet 2012;285:459-67.

Cite this article as: Niu D, Bai Y, Yao Q, Zhou L, Huang X, Zhao C. AQP2 as a diagnostic immunohistochemical marker for pheochromocytoma and/or paraganglioma. Gland Surg 2020;9(2):200-208. doi: 10.21037/gs.2020.01.19
26. Sawada T, Kato Y, Kobayashi M. Expression of aquaporine-4 in central nervous system tumors. Brain Tumor Pathol 2007;24:81-4. 